Int. J. Morphol.,

38(1):159-164, 2020.

\title{
Tubérculo Marginal del Hueso Cigomático
}

\author{
Marginal Tubercle of Zygomatic Bone
}

Juan Silva ${ }^{1,2}$; Claudia Araya ${ }^{1}$; Sebastián Pardo ${ }^{1}$; Tomás Reyes ${ }^{1}$; Andrés Salcedo ${ }^{1,3,4}$; Alvaro Sanhueza ${ }^{4}$ \& Samara Liberona $^{5}$

SILVA, J.; ARAYA, C.; PARDO, S.; REYES, T.; SALCEDO, A.; SANHUEZA, A. \& LIBERONA, S. Tubérculo marginal del hueso cigomático. Int. J. Morphol., 38(1):159-164, 2020.

RESUMEN: El hueso cigomático, ubicado en la parte superior y lateral de la cara, es un hueso par e irregular con forma cuadrilátera o romboidal. Se describen 2 caras, 4 aristas y 4 ángulos, forma cavidades, permite la inserción muscular y aponeurótica, es parte de la arquitectura facial, distribuye las fuerzas masticatorias y permite el paso del nervio cigomático. Su margen postero-superior presenta una prominencia ósea conocida como tubérculo marginal, en el que se observa la inserción de la fascia temporal. El objetivo de este trabajo fue describir las características particulares de esta prominencia. La muestra correspondió a 30 cráneos de adultos chilenos de ambos sexos. A través de una serie de puntos óseos, se describió la presencia, ubicación, tamaño, forma, cortical y trabeculado del tejido óseo del tubérculo marginal. Para realizar las mediciones se utilizaron cámara digital, compas de precisión y cáliper digital. El análisis radiográfico requirió tomografía computarizada de alta resolución. Los resultados mostraron que el tubérculo marginal del hueso cigomático es una prominencia constante, ubicada en el tercio medio del proceso frontal del hueso y que la mayoría de los individuos mostraron una forma semilunar. Su altura fluctúa entre 3 y $4 \mathrm{~mm}$, siendo más pronunciada en hombres que en mujeres. El grosor de la corteza ósea es directamente proporcional a la prominencia del tubérculo, mientras que el trabeculado esponjoso está inversamente relacionado con este último. El análisis de estos resultados parece indicar que las fuerzas biomecánicas ejercidas por la musculatura masticatoria y transmitidas por la fascia temporal, determinan la morfología externa e interna de esta prominencia y del propio hueso cigomático. Concluimos, declarando la necesidad de revisar el conocimiento anatómico a la luz de las nuevas técnicas de imagen e integración disciplinar.

PALABRAS CLAVE: Cráneo; Huesos de la cara; Fosa temporal; Hueso cigomático; Tubérculo marginal del hueso cigomático.

\section{INTRODUCCIÓN}

El hueso cigomático (HC), también conocido como hueso pómulo, hueso malar, hueso yugal o hueso de la mejilla, se describe como un hueso par, lateral e irregular. Se encuentra ubicado entre hueso frontal, hueso esfenoides, hueso temporal y hueso maxilar, uniéndolos entre sí formando el pómulo de la cara. En general, presenta una forma cuadrilátera o romboidal, con dos caras, cuatro márgenes y cuatro ángulos. Se encuentra en la parte superior de la cara determinando su límite lateral (Fig. 1a-b). Durante su desarrollo se describen tres puntos de osificación visibles al final del segundo mes de vida intrauterina y completamente unidos al quinto. Este hueso se halla compuesto principalmente por tejido óseo muy duro y compacto (Rambaud \& Renault, 1864; Sappey, 1874; Beaunis \& Bouchard, 1878; Testut et al., 1965; Rouvière \& Delmas, 1999).
Las funciones del HC descritas en la literatura son: formar cavidades comunes de cráneo y cara (fosa temporal y órbita), permitir la inserción muscular (músculos; temporal, masetero, cigomático mayor, cigomático menor), permitir la inserción aponeurótica (fascia temporal), constituir parte de los pilares y arcos de refuerzo de cráneo y cara (pilar fronto-cigomático y arco cigomático-temporal), distribuir fuerzas biomecánicas ejercidas durante la masticación y por último, permitir el paso del nervio cigomático y sus dos ramas terminales a través del conducto cigomático, hacia las regiones geniana y temporal (Cruveilhier, 1851; Sicher \& Tandler, 1930; Aprile et al., 1971; Velayos, 2001).

De los cuatro márgenes descritos en el HC, el posterosuperior presenta una forma sigmoidea característica. Esta for-

\footnotetext{
${ }^{1}$ Facultad de Medicina y Ciencia, Universidad San Sebastián, Chile.

${ }^{2}$ Departamento de Anatomía y Medicina Legal, Facultad de Medicina, Universidad de Chile, Chile.

${ }^{3}$ Facultad de Odontología, Universidad San Sebastián, Chile.

${ }^{4}$ Escuela de Odontología, Facultad de Ciencias, Universidad Mayor, Chile.

${ }^{5}$ Facultad de Ciencias para el Cuidado de la Salud, Universidad San Sebastián, Chile.
} 
ma está determinada por la presencia de una prominencia ósea ubicada algo por debajo de la sutura fronto-cigomática (Spalteholz, 1972; Castro \& Quevedo, 1984; Williams, 1998; Dauber, 2007). Esta estructura prominente se denomina tubérculo marginal (TM); su presencia, altura y extensión son muy variables, es fácilmente palpable a través de la piel y tendría por función permitir la inserción de parte del margen anterior de la fascia temporal (Calleja, 1870; Cunningham, 1949; Hamilton, 1976; Lockhart et al., 1988). En relación a su importancia clínica, se ha descrito que TM grandes podrían ser un obstáculo durante el acceso quirúrgico en la craneotomía estándar minipterional, utilizada en la exéresis de hemangiomas de ala mayor de esfenoides (Aldahak et al., 2016).

Debido a que la caracterización morfo-funcional del TM ha sido abordada en forma vaga y somera en la literatura, el objetivo de este trabajo fue describir las características particulares del TM del HC, en una muestra de cráneos secos de individuos adultos chilenos de ambos sexos. El conocimiento acabado del TM, de sus características y funciones, tiene importancia en anatomía, biomecánica mandibular, antropología, cirugía, odontología e identificación humana.

\section{MATERIAL Y MÉTODO}

La muestra correspondió a 30 cráneos secos $(60$ hemicráneos), en buen estado de conservación, de individuos adultos, chilenos, de ambos sexos (16 masculinos y 14 femeninos), pertenecientes a la osteoteca de la Facultad de Medicina y Ciencia de la Universidad San Sebastián.
En la muestra se describió la presencia, ubicación, tamaño, forma y trabeculado del tejido óseo esponjoso del TM. También se determinó su ubicación en tercios horizontales (superior, medio e inferior) con respecto al eje céfalocaudal del proceso frontal del HC. Para realizar las mediciones de tamaño y forma del TM, se definieron los siguientes puntos (Fig. 2a-b): "A" correspondió al punto más posterior del TM, "B" al punto ubicado en el margen anterior del proceso frontal del $\mathrm{HC}$ en el mismo plano transversal que el punto "A", "C" al punto más posterior de la sutura fronto-cigomática, "D" a la intersección de los procesos frontal y temporal del HC, "E" al punto más profundo del canal retromalar en el mismo plano transversal que el punto "A", y "F" al punto más profundo de la cara temporal del ala mayor del esfenoides, también en el mismo plano transversal que el punto "A".

Una vez definidos estos puntos, se procedió a realizar las siguientes mediciones (Fig. 2a): distancia "A-B", que es la medida del ancho del proceso frontal del $\mathrm{HC}$ a la altura del TM. Distancia de "C a (A-B)" en una línea perpendicular, que permitió situar el vértice del TM en alguno de los tercios horizontales (superior, intermedio o inferior). Distancia de "A a (C-D)" en una perpendicular, lo cual permitió determinar la altura del TM. Ángulo "C-A-D", que nos facilitó la evaluación de la morfología del TM. En la Figura 2b, se esquematizan los siguientes parámetros: distancia "A-F", que permitió observar la separación entre el vértice del TM y el plano constituido por la fosa temporal y la distancia entre el punto "E a (A-F)", en una perpendicular, lo cual determinó el espacio de la fosa temporal oculto por el TM.

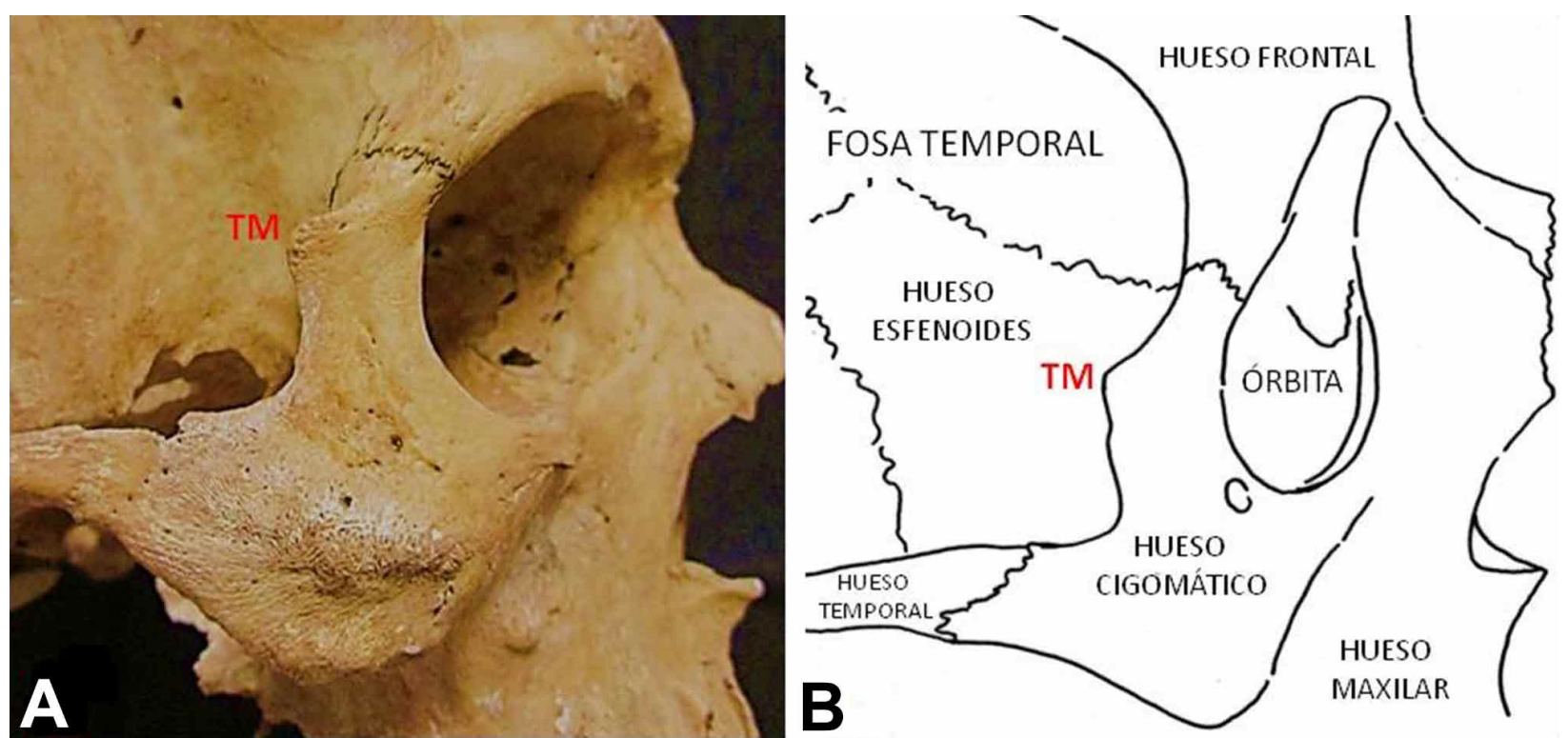

Fig. 1. En "A", fotografía en norma lateral derecha de cráneo seco, donde se puede observar un TM prominente (TM). En "B", esquema especificando las principales estructuras de la región temporal, frontal, geniana y orbita. 

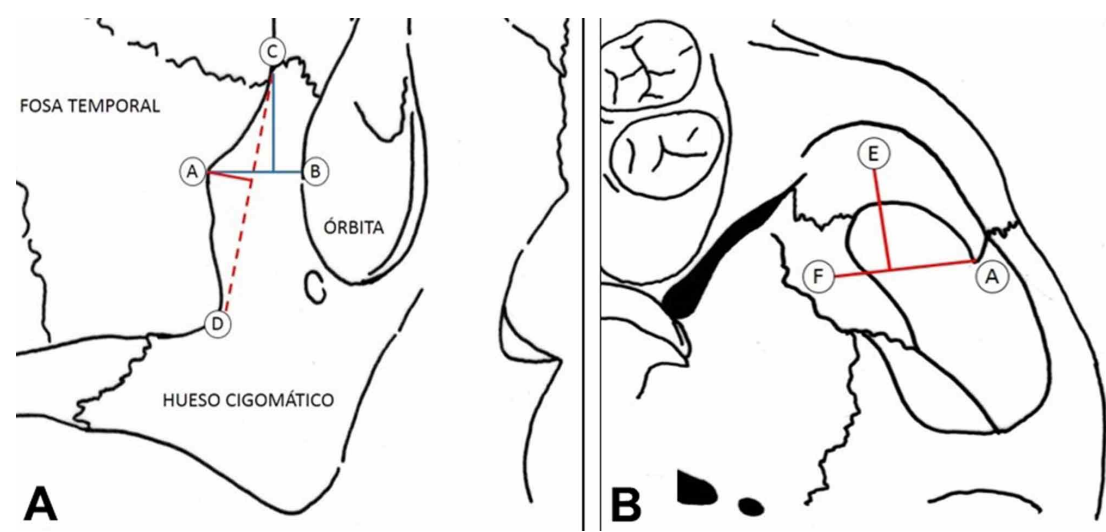

Fig. 2. Se indican las estructuras y dimensiones que fueron consideradas en el estudio. En "A", las estructuras consideradas en norma lateral. En "B", las estructuras consideradas en norma basal, específicamente a la altura de la fosa infratemporal.
Una vez dimensionado el TM, se clasificó según su forma general utilizando cuatro variables cualitativas nominales: triangular, semilunar, cuadrangular y aplanado. Asimismo, su margen se clasificó en romo, agudo, rugoso y/o liso.

Para el correcto registro de las variables de los cráneos, se fotografiaron con cámara digital marca Canon $\bigodot$ modelo PowerShot $\subset$ D30. Luego se procedió a su medición con compás de precisión marca Staedtler@, modelo Mars 552 y cáliper digital marca Mitutoyo@, serie 500 modelo 144. Todas estas mediciones fueron tomadas 3 veces y el valor registrado corresponde al promedio simple.

Finalmente, se obtuvieron tomografías computadas de alta resolución, para lo cual se utilizó equipo Planmeca Promax 3D® de la Facultad de Odontología de la Universi- dad San Sebastián, utilizando un protocolo de exposición previamente definido con parámetros de 90 Kv, 14 mAy 24 segundos de exposición. El tamaño del campo de visión (FOV) fue medio y programado hacia la rama mandibular. El cráneo (sin mandíbula) fue posicionado en una inclinación horizontal de 20 grados, pasando el indicador láser de la línea media por el margen interno de la órbita, con el objetivo de posicionar el HC en la mitad. Para la determinación de la morfología del HC y del TM, se utilizaron principalmente cortes coronales, sagitales y axiales, previo al trazado de la línea latero medial en el corte axial de manera unilateral. Respecto a la morfología interna del TM del HC, se analizaron cualitativa y descriptivamente el hueso derecho e izquierdo, consignando si presentaban o no algún signo de cambio en el trabeculado y/o densidad ósea principalmente en el corte axial y sagital.

\begin{tabular}{|c|c|c|c|c|c|}
\hline \multirow[b]{2}{*}{ Clasificación } & \multirow[b]{2}{*}{ ESQUEMA } & \multicolumn{2}{|c|}{ Derecha } & \multicolumn{2}{|c|}{ Izquierda } \\
\hline & & M \% & $\mathrm{F} \%$ & M \% & F \% \\
\hline Semilunar & & 31,25 & 42,86 & 50 & 35,71 \\
\hline Triangular & & 56,25 & 35,71 & 0 & 28,57 \\
\hline Aplanado & & 6,25 & 21,43 & 18,75 & 28,57 \\
\hline Cuadrangular & & 6,25 & 0 & 31,25 & 7,143 \\
\hline
\end{tabular}

Tabla I. resumen de los resultados obtenidos de la clasificación cualitativa nominal con respecto a la forma del TM (semilunar, triangular, aplanado y cuadrangular). Los resultados se encuentran registrados en porcentajes de representación divididos por sexo y lado. Además se ha agregado un esquema representativo de la forma del TM presente en la muestra. 


\section{RESULTADOS}

En la observación directa y a la palpación dactilar, se pudo evidenciar la presencia de TM en los 60 hemicráneos, de los cuales el 86,6\% se ubicó en el tercio medio del proceso frontal del HC y el 13,4\% restante en el tercio superior, no se logró determinar la presencia de esta prominencia en el tercio inferior del mismo proceso frontal. Los resultados de la clasificación cualitativa nominal de los TM se observan en la Tabla I. Los resultados de las mediciones de las magnitudes de las distancias entre los puntos y líneas previamente establecidos, se encuentran en la Tabla II.
Los resultados imagenológicos de la morfología interna del TC, evidenciaron que en cráneos con TM prominentes se observa una gran densidad cortical, escaso trabeculado óseo, de disposición, más bien laxa, con espacios medulares amplios y en baja cantidad (Figs. 3a y 4). En contraste con este hallazgo, en los cráneos con TM menos prominentes, se evidenció una cortical delgada, con gran trabeculado óseo denso, con espacios medulares pequeños y abundantes (Figs. 3b y 5).

Tabla II. resumen de los resultados obtenidos en las distancias descritas en el texto. Todas las medidas se registraron en $\mathrm{mm}$ (F, femenino / M, masculino).

\begin{tabular}{|c|c|c|c|c|c|c|c|}
\hline \multicolumn{8}{|c|}{ Derecha } \\
\hline & A-B & C-(A-B) & C-D & A-(C-D) & C-A-D & A-F & E-(A-F) \\
\hline $\mathbf{F}$ & 10,7 & 9,7 & 25,1 & 3,3 & 152,4 & 15,2 & 6,1 \\
\hline M & 11,7 & 9,0 & 25,7 & 4,3 & 143,6 & 15,5 & 5,0 \\
\hline \multicolumn{8}{|c|}{ Izquierda } \\
\hline & A-B & C-(A-B) & C-D & A-(C-D) & C-A-D & A-F & E-(A-F) \\
\hline $\mathbf{F}$ & 10,5 & 10,7 & 24,2 & 3,1 & 153,6 & 14,0 & 5,6 \\
\hline M & 11,5 & 10,0 & 26,0 & 3,6 & 147,4 & 15,3 & 5,0 \\
\hline
\end{tabular}
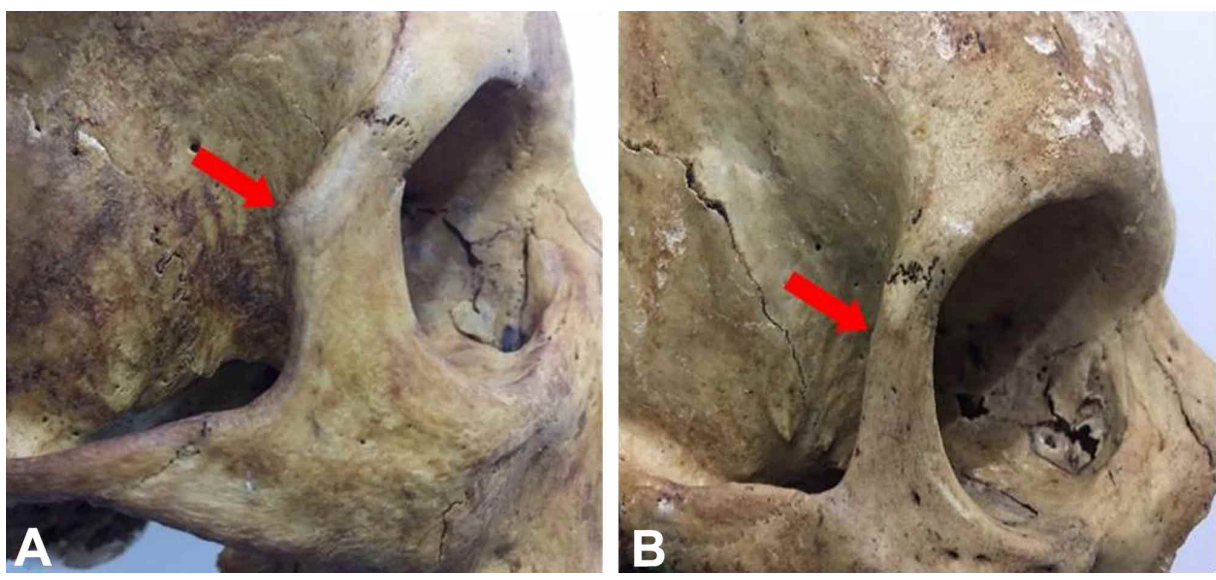

Fig. 3. En "A", fotografía en norma lateral derecha de cráneo con TM prominente y de forma triangular (flecha roja). En "B" fotografía en norma lateral derecha, de cráneo que presento TM poco prominente y forma aplanada (flecha roja).

Fig. 4. Imágenes radiográficas obtenidas del cráneo de la figura $3 \mathrm{~A}$. Este cráneo es representativo de TM prominentes. En "A" reconstrucción axial de $\mathrm{HC}$ a la altura de TM (C: cortical, TO: trabeculado óseo). en "B" su reconstrucción sagital (C: cortical, TO: trabeculado óseo). En "C" su reconstrucción coronal $(\mathrm{C}$ : cortical, TO: trabeculado óseo).
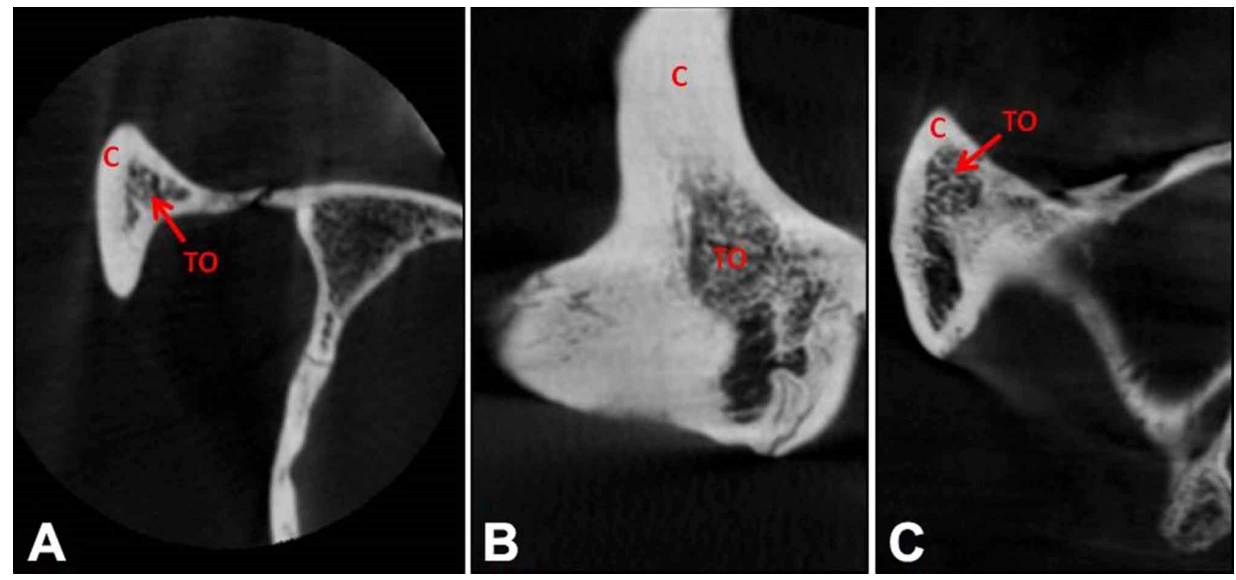

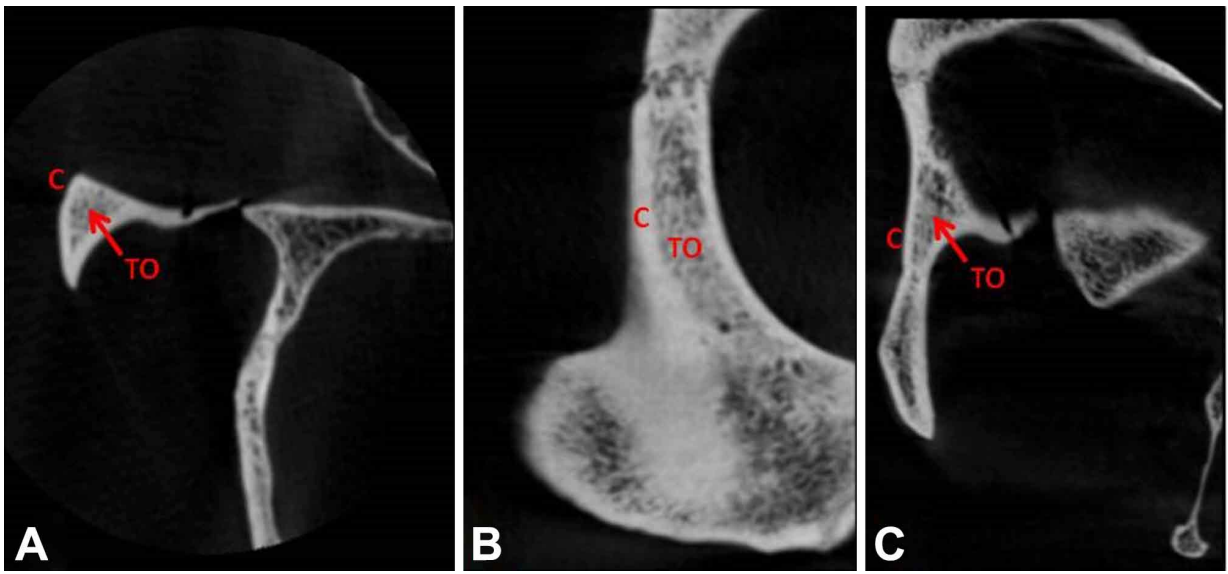

Fig. 5. Imágenes radiográficas obtenidas del cráneo de la figura 3b. Este cráneo es representativo de TM poco prominentes o aplanados. En "A" reconstrucción axial de $\mathrm{HC}$ a la altura de TM (C: cortical, TO: trabeculado óseo). en "B" su reconstrucción sagital (C: cortical, TO: trabeculado óseo). En "C" su reconstrucción coronal (C: cortical, TO: trabeculado óseo).

\section{DISCUSIÓN}

Los resultados de este trabajo indican que el TM del $\mathrm{HC}$, clásicamente descrito como estructura inconstante, se presentó en el $100 \%$ de la muestra observada, inclusive en la mayoría de los casos, se evidenció un tubérculo prominente.

El estudio radiográfico del TM, indica una morfología ósea característica de huesos que presentan inserciones musculares fuertes (mucho más que la que genera una inserción fascial).

El análisis de estos resultados parecen indicar que la fuerzas biomecánicas ejercidas por la musculatura masticatoria, que presenta inserción en la fascia temporal (músculos temporal y masetero), estaría determinando la morfología externa del TM y la arquitectura ósea interna del trabeculado interno del HC.

La puesta al día de los contenidos morfológicos se hace enormemente necesaria, no solamente por las nuevas técnicas imagenológicas con las que contamos hoy, sino también por la necesidad de integración profunda entre las disciplinas morfológicas, fisiológicas y clínicas.

\section{CONCLUSIONES}

En nuestro estudio, el TM del HC es una prominencia constante, que está ubicada en el tercio medio del proceso frontal de dicho hueso, de forma semilunar, aunque con frecuentes variaciones anatómicas. Su altura fluctúa entre los 3 y $4 \mathrm{~mm}$, siendo más pronunciado en hombres que en mujeres. El grosor de la cortical ósea es directamente proporcional a la prominencia del tubérculo, mientras que el trabeculado esponjoso se relaciona inversamente con esta última.
Finalmente, concluimos junto a Marie François Xavier Bichat (anatomista francés, 1771-1802), que "la Anatomía no es tal como se nos enseña" (Lain, 1946) y este trabajo permite evidenciar que es necesario mirar y volver a mirar con ojo crítico, los conocimientos anatómicos. Esto permitirá interpretar adecuadamente la forma y función de las estructuras del cuerpo humano, mejorando el conocimiento disciplinar y su aplicación en medicina.

SILVA, J.; ARAYA, C.; PARDO, S.; REYES, T.; SALCEDO, A.; SANHUEZA, A. \& LIBERONA, S. Marginal tubercle of zygomatic bone. Int. J. Morphol., 38(1):159-164, 2020.

SUMMARY: The zygomatic bone, located in the upper and lateral area of the face, is an even and irregular quadrilateral or rhomboid shaped bone. It presents 2 faces, 4 margins and 4 angles. It forms cavities, allows muscular and aponeurotic insertion, is part of the facial architecture, distributes masticatory forces and allows the passage of the zygomatic nerve. Its postero-superior margin presents a bony prominence known as a marginal tubercle, in which the insertion of the temporal fascia is observed. The objective of this work was to describe the particular characteristics of this prominence. The sample corresponded to 30 skulls of Chilean adults of both sexes. Through a series of bone points, the presence, location, size, shape, cortical and trabeculate of the bone tissue of the marginal tubercle was described. A digital camera, precision compass and digital caliper were used to perform the measurements. The radiographic analysis required high-resolution computed tomography. The results showed that the marginal tubercle of the zygomatic bone is a constant prominence, located in the middle third of the frontal process of the bone and that most individuals showed a semilunar shape. Its height fluctuated between 3 and 4 $\mathrm{mm}$, being more pronounced in men than in women. The thickness of the bone cortex was directly proportional to the prominence of the tuber, while the spongy trabeculate was inversely related to the latter. The analysis of these results seems to indicate that the 
biomechanical forces exerted by the masticatory musculature and transmitted by the temporal fascia, determine the external and internal morphology of this prominence, and of the zygomatic bone itself. In conclusion, it is recommended to review anatomical knowledge in the light of new imaging techniques and disciplinary integration.

KEY WORDS: Skull; Facial bones; Temporal fossa; Zygomatic bone; Marginal tubercle of the zygomatic bone.

\section{REFERENCIAS BIBLIOGRÁFICAS}

Aldahak, N.; El Tantowy, M.; Dupre, D.; Yu, A.; Keller, J. T.; Froelich, S. \& Aziz, K. M. Drilling of the marginal tubercle to enhance exposure via mini pterional approach: An anatomical study and clinical series of 25 sphenoid wing meningiomas. Surg. Neurol. Int., 7(Suppl. 40):S989S994, 2016

Aprile, H.; Figún, M. \& Garino, R. Anatomía Odontológica Orocervicofacial. Buenos Aires, Editorial el Ateneo, 1971.

Beaunis, H. \& Bouchard, A. Nuevos elementos de anatomía descriptiva y de embriología. $2^{\mathrm{a}}$ ed. Madrid, Moya y Plaza, 1878.

Calleja, J. Tratado de Anatomía Humana. Valladolid, Editorial de los Hijos de Rodriguez, 1870.

Castro, M. \& Quevedo, S. Proposiciones metodológicas para el estudio de los rasgos no métricos en el cráneo humano. Bol. Mus. Nac. Hist. Nat. Chile, 40:173-210, 1984

Cruveilhier, J. Traité d'Anatomie Descriptive. Paris, Editorial Labé, 1851.

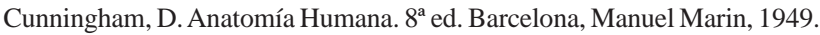

Dauber, W. Feneis. Nomenclatura Anatómica Ilustrada. $5^{\mathrm{a}}$ ed. Barcelona, Elsevier-Masson, 2007.

Hamilton, W. Textbook of Human Anatomy. $2^{\mathrm{a}}$ ed. Saint Louis, Mosby, 1976.

Lain, P. Clásicos de la Medicina, Bichat. Madrid, Ediciones El Centenario, 1946.

Lockhart, R.; Hamilton, G. \& Fyfe, F. Anatomía Humana. Ciudad de México, Interamericana, 1988.

Rambaud, A. \& Renault, C. Origine et Développement des Os. Paris, F. Chamerot, 1864.

Rouvière, H. \& Delmas, A. Anatomía Humana Descriptiva, Topográfica y Funcional. $10^{\mathrm{a}}$ ed. Barcelona, Masson, 1999.

Sappey, P. Tratado de Anatomía Descriptiva. $2^{\text {a }}$ ed. Madrid, Carlos BaillyBailliere, 1874.

Sicher, H. \& Tandler, J. Anatomía para Dentistas. Barcelona, Labor, 1930. Spalteholz, W. Atlas de Anatomía Humana. 6ª ed. Barcelona, Labor, 1972.

Testut, L.; Latarjet, A. \& Latarjet, M. Tratado de Anatomía Humana. $9^{\mathrm{a}}$ ed. Barcelona, Salvat Editores, 1965.

Velayos, J. Anatomía de la Cabeza. $3^{\mathrm{a}}$ ed. Madrid, Médica Panamericana, 2001.

Williams, P. L. Anatomía de Gray. 38ª ed. Madrid, Harcourt Brace, 1998.
Dirección para correspondencia:

Prof. Dr. Juan Luis Silva Rubilar

Facultad de Medicina y Ciencia

Universidad San Sebastián

Lota 2465. C. P. 7510157

Providencia

Santiago

CHILE

Email: juan.silva@uss.cl

Recibido : 21-08-2019

Aceptado: 05-09-2019 